\title{
Synthesis of Gold Nanoparticle Using Coconut for Encapsulation of Curcumin
}

\author{
KARL SHAINE MANALILI PEREZ ${ }^{1 *}$ and MARIO ABESAMIS JR. ${ }^{1}$ \\ 'Department of Chemistry and Environmental Science, College of Arts and Sciences, Nueva Ecija \\ University of Science and Technology, Cabanatuan City, Nueva Ecija, 3100, Philippines. \\ *Corresponding author E-mail: karlshaineperez@gmail.com
}

http://dx.doi.org/10.13005/ojc/370115

(Received: December 02, 2020; Accepted: February 10, 2021)

\section{ABSTRACT}

Today, cancer is one of the leading causes of death in the world. Currently, there are no cancer treating-drugs available. Cancer cells generally respond to radical-scavenging compounds like polyphenols, and Curcumin is one of them. Also known as diferuloylmethane, Curcumin is a low molecular-weight active constituent from the perennial herb Curcuma longa (commonly known as turmeric) with an established anticancer property. However, this compound has very low bioavailability for efficient absorption. In the present study, Curcumin is encapsulated with gold nanoparticles to improve its bioavailability. Gold nanoparticle synthesized from coconut extract renders the element to be less toxic. The curcumin-gold nanoparticle solution was characterized using Scanning Electron Microscope for its morphology and LC ${ }_{50}$ (Lethal Concentration needed to kill the $50 \%$ of the population) toxicity of the solution. The results confirmed the synthesis and encapsulation of Curcumin with gold nanoparticles.

Keywords: Gold nanoparticles, Curcumin, Encapsulation, Anticancer property, Coconut extract.

\section{INTRODUCTION}

Cancer is one of the top causes of demise in the world. About 12.7 million cancer cases and 7.6 million people died of cancer in the world in $2008^{1}$.

Curcumin is a compound that shown anticancer effects in recent studies which includes inhibiting carcinogenesis, angiogenesis, and also prevention of the growth of tumor ${ }^{2}$.

Curcumin, with its numerous medicinal potentials, researchers still struggle to develop this substance into a functional medicine due to its low oral bioavailability. Due to extensive intestinal and hepatic metabolism, the researchers observed low levels of Curcumin in the serum and tissue ${ }^{3}$. To solve this problem, researchers added piperine to the formulation. Piperine was known for its intestinal and hepatic metabolism inhibition which increases the bioavailability of Curcumin by $2000 \%$, but piperine may cause toxicity in people taking specific drugs ${ }^{4}$.

Gold nanoparticles (AuNPs) have various properties, including unique electronic properties and a highly multifunctional platform that can be

This is an Open Access article licensed under a Creative Commons license: Attribution 4.0 International (CC- BY). Published by Oriental Scientific Publishing Company @ 2018

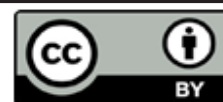


used for diagnosing diseases and can be used for selective drug delivery agents. Gold nanoparticles have the potential to increase the bioavailability of Curcumin through selectively delivery ${ }^{5}$.

The study attempts to encapsulate Curcumin using gold nanoparticles synthesized using Cocos nucifera extract to improve its oral bioavailability and determine its cytotoxicity.

\section{MATERIALS AND METHODS}

\section{Sample Collection}

The coconut was collected from the local market in Cabanatuan city. The analytical grade tetrachloroauric acid was purchased from Sigma Aldrich, and no further treatment was applied. The Curcumin was purchased from Shaanxi Sangherb Biotech Inc, and the zebrafish from Central Luzon State University.

\section{Sample Preparation}

The coir of the coconut was washed and dried in the air. A $10 \mathrm{~g}$ of dried coconut-coir was mixed with $100 \mathrm{~mL}$ water and allowed to stand for 24 hours, and then filtered through a cheesecloth.

\section{Synthesis of Gold Nanoparticles using Coconut Extract}

This method was adopted from the study of Paul et al.,(2013) and was modified according to Cabrera et al.,(2017). About $0.5 \mathrm{~g}$ of tetrachloroauric acid salt was used to prepare a $100 \mathrm{mM}$ solution. To make one $\mathrm{mM}$ of tetrachloroauric acid salt. An aliquot of five hundred microliters was diluted to 50 milliliters. A $100 \mathrm{~mL}$ tetrachloroauric salt solution in an Erlenmeyer flask was boiled with continuous stirring. About 10 milliliters of the coconut extract was added directly into the boiled solution. The mixture was cooled at room temperature.

\section{Characterization of Synthesized Gold Nanoparticle}

The synthesized gold nanoparticle was subjected to UV-Vis Spectroscopy to confirm its synthesis.

\section{Encapsulation of Curcumin using Gold Nanoparticle For conjugation of Curcumin, $50 \mathrm{mg}$ of crystalline Curcumin was dissolved in $25 \mathrm{~mL}$ acetone, and this solution was added to the $100 \mathrm{~mL}$ of gold nanoparticles solution with stirring. Further, this mixture was stirred for three hours at $60^{\circ} \mathrm{C}$ and then cooled down to room temperature. The}

Au-curcumin solution was centrifuged at $4000 \mathrm{rpm}$ to remove unattached Curcumin. This was done three times to ensure that no free curcumin molecules are left in the final conjugate.

\section{Characterization of the Encapsulated Curcumin}

The curcumin-gold nanoparticle solution was characterized using Scanning Electron Microscopy analysis to determine its size.

\section{Encapsulation Efficiency of the Encapsulated Curcumin}

The resulting solution of Au-curcumin was subjected to spectrophotometric analysis at $426 \mathrm{~nm}$. The concentration was compared to the original curcumin concentration. The encapsulation efficiency (EE) of the samples were calculated with the following equation:

$\mathrm{EE}(\%)=((\mathrm{Y}) \mathrm{X100}) /(\mathrm{Z})$

$\mathrm{Y}=$ concentration of curcumin in the Au-curcumin solution

$\mathrm{Z}=$ concentration of original curcumin

\section{Determination of $\mathrm{LC}_{50}$}

The gold nanoparticle encapsulated Curcumin was fed to the zebrafish and observed after $48 \mathrm{~h}$ to determine the $\mathrm{LC}_{50}$ of the Gold Nanoparticle Encapsulated Curcumin by assessing the mortality of zebrafish against the encapsulated Curcumin. Probit analysis was used to determine the lethal concentration to kill 50 percent of the zebrafish.

\section{RESULTS AND DISCUSSIONS}

\section{Synthesis of gold nanoparticle using coconut extract}

The synthesized gold nanoparticle was brick-red in color. As shown in Fig. 1, the synthesized gold nanoparticle (AuNP) solution was brick red, along with the use of a laser pointer, which confirmed the presence of AuNP.

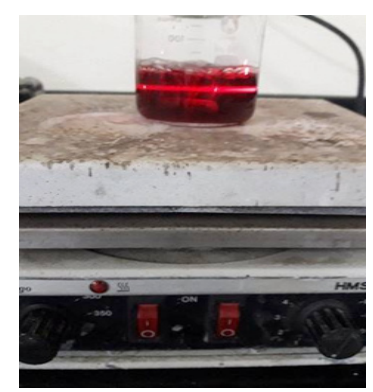

Fig. 1. Synthesized gold nanoparticles 


\section{Confirmation of Gold Nanoparticle}

The Synthesis of the Gold Nanoparticle was confirmed using a UV-Vis spectrophotometer, and the spectra are shown in Figure 2.

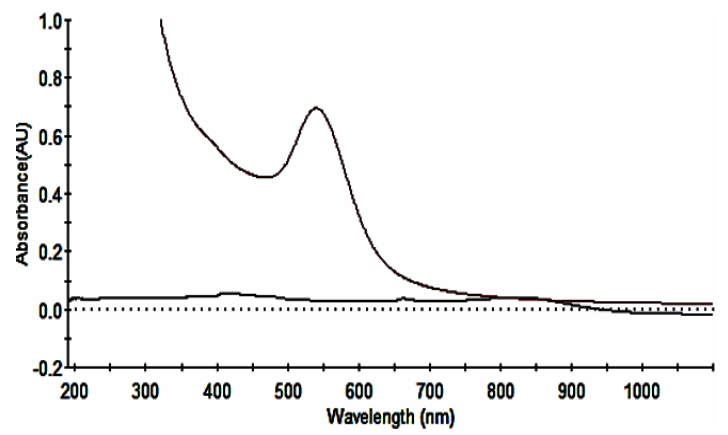

Fig. 2. UV-Vis Spectra of Gold Nanoparticle

Encapsulation of Curcumin with Gold Nanoparticle

The synthesized gold nanoparticle solution was mixed with the curcumin to make the complex as shown in the figure below. The presence of the orange color confirms the attachment of the curcumin with the gold nanoparticle.

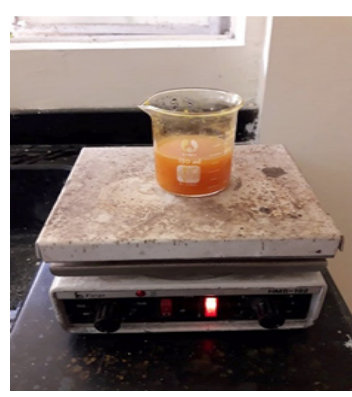

Fig. 3. Au-curcumin solution

\section{Encapsulation Efficiency of Curcumin}

The concentration of Curcumin in the solution was determined using UV-Vis spectroscopy, and the standard curve of Curcumin with the regression of 0.9775 was used to determine its concentration. Table 1 shows the absorbance of each sample.

Table 1: UV-Vis absorbance of Au-curcumin and curcumin

\begin{tabular}{lccc}
\hline Name of sample & Replicate 1 & Replicate 2 & Replicate 3 \\
\hline $\begin{array}{l}\text { Au-curcumin } \\
\text { Curcumin }\end{array}$ & 0.7698 & 0.7364 & 0.7662 \\
& 0.1059 & 0.0953 & 0.1177 \\
\hline
\end{tabular}

\section{Size of gold nanoparticle-curcumin}

The gold nanoparticle size was determined using Scanning Electron Microscopy at the De La Salle University, Physics Laboratory. The magnification of the SEM was 50,000 .

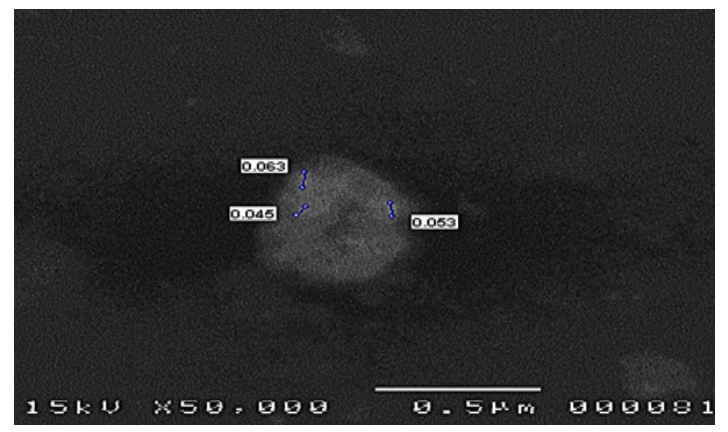

Fig. 4. Scanning Electron Microscopy of Au-Curcumin

Toxicity assay Lethal Concentration to kill $\mathbf{5 0} \%$ of the population $\left(\mathrm{LC}_{50}\right)$

The $\mathrm{LC}_{50}$ of Au-curcumin was determined using the toxicity assay using zebrafish; the mortality was presented in the table below.

Table 2: Probit Analysis for the LC $_{50}$

\begin{tabular}{ccccccccc}
\hline Concentration R1 R2 Conc. total mort \%mort & $\begin{array}{c}\text { log } \\
\text { (conc.) }\end{array}$ & probit \\
\hline $50 \%$ & 10 & 10 & 500 & 20 & 20 & 100 & 2.69897 & 8.1 \\
$10 \%$ & 4 & 4 & 100 & 20 & 8 & 40 & 2 & 4.75 \\
$5 \%$ & 3 & 4 & 50 & 20 & 7 & 35 & 1.69897 & 4.61 \\
$1 \%$ & 2 & 3 & 10 & 20 & 5 & 25 & 1 & 4.33 \\
$0.50 \%$ & 1 & 2 & 5 & 20 & 3 & 15 & 0.69897 & 3.96 \\
\hline
\end{tabular}

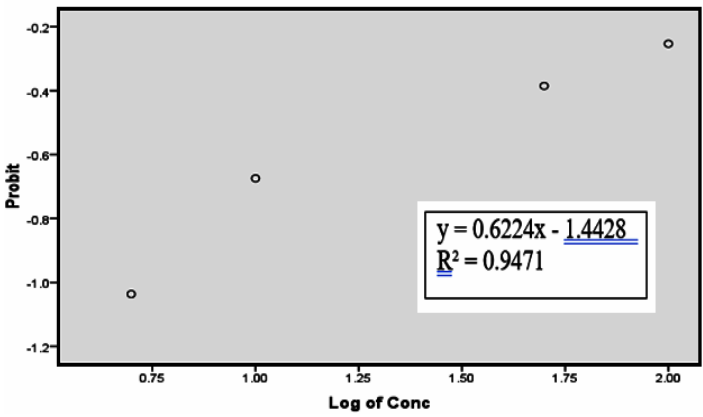

Fig. 5. Probit Transform Response of Au-curcumin solution

DISCUSSION

\section{Confirmation of Gold Nanoparticle}

The gold nanoparticle exhibits absorbance of 0.695887 at the wavelength of $540 \mathrm{~nm}$. According to the study of Moustaoui et al.,(2019), gold nanoparticles exhibit an absorbance peak ranging from $500-700 \mathrm{~nm}$ depending on their size from $10 \mathrm{~nm}$ up to $60 \mathrm{~nm}$.

\section{Encapsulation Efficiency of Curcumin}

According to the result, the Au-curcumin has an average absorbance of 0.7575 and the untreated Curcumin has an average absorbance of 0.1063 . 
Using the Curcumin standard the concentration of Curcumin in the au-curcumin solution was determined to have $0.8735 \mathrm{ppm}$, and the concentration of the original solution was $2.4315 \mathrm{ppm}$. And the computed encapsulation efficiency was $35.92 \%$.

\section{Size of gold nanoparticle-curcumin}

The gold nanoparticle in the Au-curcumin complex exhibited an average size of $54 \mathrm{~nm}$. The typical size of nanoparticle that is used for medical purposes is $\leq 100 \mathrm{~nm}^{9}$. The size of the nanoparticle is important depending on its purpose, for this study gold nanoparticle that needed to be synthesized must have small size but not too small that it will have small surface area that will lead to the faster release of the drug ${ }^{10}$. The modification done to the method of Cabrera et al.,(2017) increases the size from $21.62 \mathrm{~nm}$ to $54 \mathrm{~nm}$.

\section{Toxicity assay Lethal Concentration to kill $\mathbf{5 0 \%}$ of the population $\left(\mathrm{LC}_{50}\right)$}

Based on the probit analysis done to the zebrafish. The lethal concentration to kill 50 percent of the population is $61.97 \mathrm{ppm}$ of Au-curcumin solution. The $\mathrm{LC}_{50}$ suggested that the synthesized
Au-curcumin was not generally toxic than the other heavy metals because it takes about $62 \mathrm{ppm}$ to damage a living organism compared to mercury with 0.0005 ppm.

\section{CONCLUSION}

The synthesis of the Au-curcumin solution was successful with a $35.92 \%$ encapsulation efficiency and an average size of $54 \mathrm{~nm}$ which is small enough to be an efficient drug delivery compound and big enough to control the release of the drug ${ }^{10}$. The Solution was found to be less lethal compared to other metal compounds which makes it suitable for drug delivery.

\section{ACKNOWLEDGEMENT}

We want to acknowledge Ma'am Danilet Vi M. Mendoza, Sir Darwin F. Reyes, Sir Gil Felicisimo Cabrera and Noel Coloma for their support and advice. Thank you.

\section{Conflict of Interest}

There is no conflict of interest in this paper.

\section{REFERENCES}

1. Jemal, A.; Bray, F.; Center, M. M.; Ferlay, J.; Ward, E.; and Forman, D. Influence of piperine on the pharmacokinetics of Curcumin in animals and human volunteers. Planta medica., 2011, 61(2), 69-90.

2. Mansouri, K.; Rasoulpoor, S.; Daneshkhah, A.; Abolfathi, S.; Salari, N.; Mohammadi, M., Rasoulpoor S.; Shabani, S. Clinical effects of curcumin in enhancing cancer therapy: A systematic review. BMC Cancer., 2020, 20(1).

3. Konatham, S.; Reddy, B.; Aukunuru, J. Enhanced liver delivery and sustained release of Curcumin with drug loaded nanoparticles after intravenous administration in rats, $A J$ Pharm Res Health Care., 2010, 3(4), 99-108.

4. Shoba, G.; Joy, D.; Joseph, T.; Majeed, M.; Rajendran, R.; Srinivas, P. S. Influence of Piperine on the Pharmacokinetics of Curcumin in Animals and Human Volunteers. Planta Medica., 1998, 64, 353-356.

5. Dreaden, E. C.; Austin, L. A.; Mackey, M. A.; ElSayed, M. A. Size matters: gold nanoparticles in targeted cancer drug delivery. Therapeutic delivery., 2012, 3(4), 457-478.

6. Paul, K.; Bag, B.G.; Samanta, K. Green coconut (Cocos nucifera Linn) shell extract mediated size controlled green synthesis of polyshaped gold nanoparticles and its application in catalysis. Appl Nanosci., 2014, 4, 769-775.

7. Cabrera, G. F.; Balbin, M.; Eugenio, P. J.; Zapanta, C.; Monserate, J.; Salazar, J.; Mingala, C. Green synthesis of gold nanoparticles reduced and stabilized by sodium glutamate and sodium dodecyl sulfate. Biochemical and Biophysical Research Communications., 2017, 484(4), 774-780.

8. Moustaoui, H.; Saber, J.; Djeddi, I.; Liu, Q.; Diallo, A. T.; Spadavecchia, J.; Djaker, N. Shape and size effect on photothermal heat elevation of gold nanoparticles: Absorption coefficient experimental measurement of spherical and urchin-shaped gold nanoparticles. The Journal of Physical Chemistry C., 2019, 123(28), 17548-17554.

9. Murthy, S. K. Nanoparticles in modern medicine: state of the art and future challenges. International journal of nanomedicine., 2007, 2(2), 129-141.

10. Rizvi, S. A. A.; Saleh, A. M. Applications of nanoparticle systems in drug delivery technology. Saudi Pharmaceutical Journal., 2018, 26(1), 64-70. 